\title{
Feelings of control: Contingency determines experience of action
}

Authors: James W. Moore, David Lagnado*, Darvany C. Deal, \& Patrick Haggard*

Correspondence address:

James Moore

Institute of Cognitive Neuroscience

17 Queen Square

London WC1N 3AR

Tel: 02076791128

Email: j.w.moore@ucl.ac.uk

*Authors contributed equally to this study.

Acknowledgements: We thank Louise Glanville for help with testing. JM was supported

by an interdisciplinary ESRC/ MRC studentship. This research was also supported by ESRC Grant RES00231571, and by a Royal Society Research Fellowship 512009.k501/SJC/je to PH.

Accepted in press article - Cognition (uncorrected proof)

Word count: 2,896 words (Excluding figures, tables and references) 
The experience of causation is a pervasive product of the human mind. Moreover, the experience of causing an event alters subjective time: actions are perceived as temporally shifted towards their effects (Haggard, Clark, \& Kalogeras, 2002). This temporal shift depends partly on advance prediction of the effects of action, and partly on inferential "postdictive" explanations of sensory effects of action. We investigated whether a single factor of statistical contingency could explain both these aspects of causal experience. We studied the time at which people perceived a simple manual action to occur, when statistical contingency indicated a causal relation between action and effect, and when no such relation was indicated. Both predictive and inferential "postdictive" shifts in the time of action depended on strong contingency between action and effect. The experience of agency involves a process of causal learning based on statistical contingency.

\section{Introduction}

The evolution of human intelligence has allowed not just deeper understanding of the world, but a greater capacity to act on it. Such operant actions imply the ability to know that one is performing an action, and to represent its consequences (Dickinson \& Balleine, 2000). Research on the epistemic content and conscious experience of action has identified two distinct processes underlying this ability.

According to ideomotor theories (e.g. James, 1890), actions are internally represented by reference to their external consequences. On this view, making an operant action involves a prediction of the action goal, an idea supported by recent models of 
computational motor control (Blakemore, Wolpert, \& Frith, 2002; Berti et al, 2006). Alternatively, the conscious experience of operant action may be inferred from sensory evidence (Wegner, 2002). In particular, spatial and temporal correlations between thoughts, physical movement, and external events may lead to us to infer that we have caused an external event.

Voluntary actions have strong effects on the subjective passage of time (Haggard, Clark, $\&$ Kalogeras, 2002). Temporal effects provide a common measure allowing the predictive and inferential contributions to experience of action to be compared directly. When a voluntary action, but not an involuntary movement, is followed by an external event, people perceive the action as shifted in time towards its effect, and the effect as shifted earlier in time towards the action that caused it (Haggard, Clark, \& Kalogeras, 2002). This 'intentional binding' involves a predictive element, because omitting the effect does not prevent the shift in perceived time of action, as long as the probability of an effect given an action is sufficiently high (Moore \& Haggard, 2008). It also involves an inferential "postdictive" element, because the tone's occurrence shifts the perceived time of action, even when tone probability is low (Moore \& Haggard, 2008).

This suggests that the human mind builds internal models of action-effect relations, which determine the experience of action. Here, we investigate whether rules thought to govern causal learning in animals might also underlie the experience of agency in humans. Contingency is an index of the causal relation between events, and predicts patterns of operant learning in animals (Hammond, 1980). Contingency is defined here 
as the probability of an effect (E) given an action (A), minus the probability of $E$ in the absence of A. This index is known as $\Delta \mathrm{p}$ (Allan, 1980):

$$
\Delta \mathrm{p}=\mathrm{P}(\mathrm{E} \mid \mathrm{A})-\mathrm{P}(\mathrm{E} \mid \sim \mathrm{A})
$$

Where $\Delta \mathrm{p}>0$ the effect is more probable in the presence than in the absence of action. Conversely, where $\Delta \mathrm{p}<0$ the effect is less probable in the presence than in the absence of action. Contingency underlies performance of goal-directed action and explicit causal judgements (Shanks \& Dickinson, 1991), but its role in conscious experience of action itself has not been investigated. The $\Delta \mathrm{p}$ measure represents the statistical relation between actions and their effects. To calculate this relation, one needs to take into account what happens in both the presence and absence of voluntary action. Traditional epistemology holds that humans know about their own voluntary actions directly, and in a self-intimating way (Descartes, 1641/1979; Libet, Gleason, Wright, \& Pearl, 1983). On this view, consciousness of action is a corollary of the motor processes engaged during action programming. In contrast, if $\Delta \mathrm{p}$ influences action awareness, this would suggest that the experience of any individual action depends on a causal model built up through extensive background knowledge acquired during both the presence and absence of voluntary movement.

We performed an experiment to assess whether the conscious experience of action depended on the background contextual understanding of regularities in the external world, as expressed by $\Delta \mathrm{p}$, or only on immediate processing in the motor system. If 
background contextual understanding contributes to action experience, then conditions with high $\Delta \mathrm{p}$ should induce strong binding. Conversely, if immediate processing of the motor system alone determines action experience, then binding should be insensitive to changes in $\Delta \mathrm{p}$.

\section{Method}

\section{Participants}

38 participants ( 21 Female; mean age of 26 years) took part in the experiment, which lasted approximately 1 hour.

\section{Procedure}

Participants chose on each trial whether or not to press a key with their right index finger. The element of choice was included because computing $\Delta \mathrm{p}$ requires trials with and without actions. They viewed a rotating clock hand (period $2560 \mathrm{~ms}$, see Libet, Gleason, Wright, \& Pearl, 1983), and used this to judge the time of their actions (Wundt, 1908). If they decided to press, they did so at a time of their choosing within the first revolution of a clock hand. If they decided not to press they simply remained still until the end of the trial. At the end of the trial participants entered the clock time at which they pressed the key, or a dummy value if they had not pressed. Participants were asked to try to ensure that roughly half of the trials involved key presses.

A tone could also occur on each trial. If the participant acted, a tone could follow $250 \mathrm{~ms}$ later. A tone could also occur if they did not act. In this case, it occurred at a random 
time within the second revolution of the clock hand. The probability of the tone given the action and the probability of the tone in the absence of action both varied across experimental conditions (see Table 1 for details).

Table 1. Mixed factorial design used in the study.

\begin{tabular}{c|c|c|c|}
\hline \multicolumn{2}{|c|}{} & \multicolumn{2}{c|}{ Between subjects factor } \\
\hline \multirow{2}{*}{ Within subjects } & Contingent & Low tone-probability & High tone-probability \\
& $\Delta p=0.5$ & $P(T \mid A)=0.5$ & group \\
factor & & $P(T \mid \sim A)=0$ & $P(T \mid A)=0.75$ \\
& Non-contingent & $P(T \mid A)=0.5$ & $P(T \mid \sim A)=0.25$ \\
\cline { 2 - 4 } & $\Delta p=0$ & $P(T \mid \sim A)=0.5$ & $P(T \mid A)=0.75$ \\
& & & $P(T \mid \sim A)=0.75$ \\
\hline
\end{tabular}

Participants were randomly assigned to one of two tone-probability groups (19 participants in each). For the low tone-probability group, the probability of the tone in the presence of action was 0.5 . For the high tone-probability group, probability of the tone in the presence of action was 0.75 . Both groups performed two experimental conditions. In the contingent condition $\Delta \mathrm{p}$ was 0.5 , and in the non-contingent condition $\Delta \mathrm{p}$ was 0 (Table 1). Thus, although the groups differed in terms of the probability of the tone given action, the manipulation of contingency defined by $\Delta \mathrm{p}$ was equal in both (see Figure 1 for details of trial structure in each condition). 


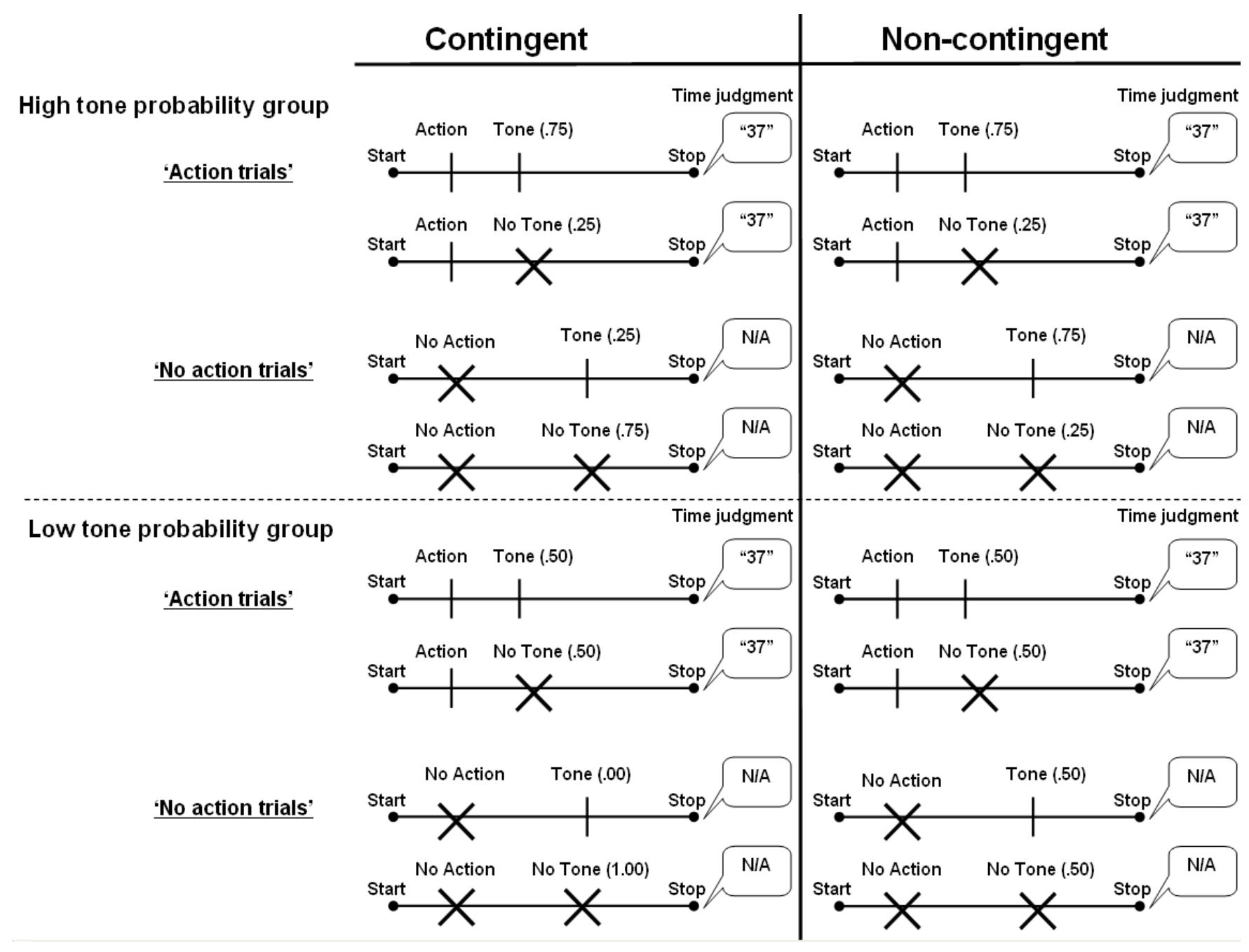

Figure 1. Trial types and structure for each level of the experimental design.

Within each condition, trials in which the participant decided to press the key were coded as 'action only' or 'action + tone'. By analysing these separately we estimated the predictive and the postdictive inferential components of the conscious experience of action. If an action is not followed by a tone, but the probability of tone given action is high, the experience of action may be influenced by the prediction that the tone might occur (Moore \& Haggard, 2008). Conversely, if any particular action is followed by a tone, action experience may be retrospectively altered by occurrence of the tone. This 
postdictive inferential process would be absent on trials where the tone does not occur. We investigated the sensitivity of both predictive and inferential processes to contingency, by independently manipulating the probability of tones given action and the probability of tones given no action.

Participants in each tone-probability group completed 3 blocks of 40 trials each in the contingent condition and 3 blocks in the non-contingent condition. Ordering of contingent/non-contingent blocks was randomised for each participant. Participants also completed two baseline blocks of 20 trials each, at the start and end of the session. In baseline blocks, participants pressed the key at a time of their choosing within the first revolution of the clock hand. Participants pressed the key on every trial, and there were no tones. The baseline conditions control for individual differences in timing judgements. To determine the binding effect between actions and tones, each participant's average error in judging the time of action in the baseline condition was subtracted from their average error in each experimental condition. A positive value indicates a shift in the experience of action towards the following tone.

On those trials where actions were followed by tones, we predicted a shift in the awareness of action towards the tone. To isolate purely postdictive inferential components of action experience, we focus on 'action + tone' trials in the low tone probability group, because the low tone probability reduces the risk of confounds due to prediction (Moore \& Haggard 2008). If background contextual knowledge affects this postdictive inferential component of action experience, this shift should be greater in this 
low tone probability group where $\Delta \mathrm{p}$ is high, since high $\Delta \mathrm{p}$ values provide strong evidence of a causal link between action and tone.

In the high tone-probability group only, we expected an additional predictive shift in the experience of action towards the tone. This should occur even on those 'action only' trials where actions were not followed by tones. If this predictive process also depends on background contextual understanding, then the predictive shift should also be greater in the contingent condition, when $\Delta \mathrm{p}$ was high.

\section{Results}

The overall percentage of trials where participants pressed the key was $62.4 \%$.

Baseline judgements (see supplementary information) for each group were compared before and after the experiment using 2x2 ANOVA. Baseline judgements were earlier after the experiment than before $(F 1,36)=5.73, p=.022)$. Baseline judgements were earlier in the high tone probability group than in the Low tone probability group $(F(1,36)$ $=4.74, p=.036)$. Since these main effects did not interact $(F(1,36)=1.35, p=.253)$, the pre and post baseline judgements were simply averaged.

Mean binding effects for actions are shown in Table 2 In the low tone-probability group, a shift in the experience of action towards the tone was only found in contingent blocks, and only for 'action + tone' trials. No shift was found for 'action only' trials, confirming that experience of action was not based on predicting the tone in this group of 
participants. Conversely, in the high tone-probability group, a delay in the awareness of action was found on both 'action only' and 'action + tone'. This confirms that their experience of action involved predicting the effects of action. To identify the contribution of contingency $(\Delta \mathrm{p})$ to inferential and predictive components of action experience respectively, we subtracted the shifts in the non-contingent condition for each subject from their shift in the contingent condition. This gives a direct measure of the effect on action experience of a 0.5 increase in $\Delta p$, achieved only by varying the number of trials with tones in the absence of action. The contingency effects are shown in Figure 2 (see also, Table 2).

Table 2. Timing judgments for experimental conditions. Mean shift in action experience towards tone in ms (SE across participants).

\begin{tabular}{ccccc}
\hline & Shift in action & Shift in action & Mean contingency- \\
Trial type & awareness $(\mathrm{ms}):$ & awareness $(\mathrm{ms}):$ & dependent shift in \\
& Non contingent & Contingent & action awareness \\
& condition $(\Delta \mathrm{p}=0)$ & condition $(\Delta \mathrm{p}=0.5)$ & $(\mathrm{ms})$
\end{tabular}

Tone-Probability Group

Low tone-probability

Action only

$-4(7)$

$-8(7)$

$-4(7)$

Action + tone

$-5(8)$

$8(8)$

$+13(5)$

High tone-probability

Action only

7 (9)

$22(7)$

$+15(7)$

Action + tone

$13(8)$

$18(8)$

$+5(5)$ 
The effects of contingency on action experience were analysed using Tone-probability group (high vs. low) as a between-subjects factor, and Trial type ('Action Only' vs. 'Action + Tone') as a within-subject factor. There were no significant main effects of Tone-probability group, $\mathrm{F}(1,36)=.52, \mathrm{p}=.47$, or Trial type, $\mathrm{F}(1,36)=.51, \mathrm{p}=.48$. However, these factors interacted significantly $\mathrm{F}(1,36)=6.31, \mathrm{p}=.017$. This arose because greater contingency increased binding in the low tone-probability group only on those trials when the tone actually occurred ('action + tone': $\mathrm{t}(18)=3.18, \mathrm{p}=.005)$, but not on those 'action only' trials where no tone occurred: $\mathrm{t}(18)=-.49, \mathrm{p}=.63$ ). That is, the postdictive inference-based shift in action experience of action due to the tone occurring was boosted by contingency.

Conversely, in the High tone-probability group greater contingency increased binding on those trials where the tone was predictable but did not in fact occur (contingency effect for 'action only' trials $\mathrm{t}(18)=2.16, \mathrm{p}=.045)$. This shift in action experience must be due to prediction of the tone rather than a retrospective inference, since no tone actually occurred. Thus, the predictive component of action experience is also sensitive to contingency. Interestingly, contingency had a smaller and non-significant effect on shift in action experience on trials when the tone actually occurred ('action + tone': $\mathrm{t}(18)=$ $.89, \mathrm{p}=.39)$. 


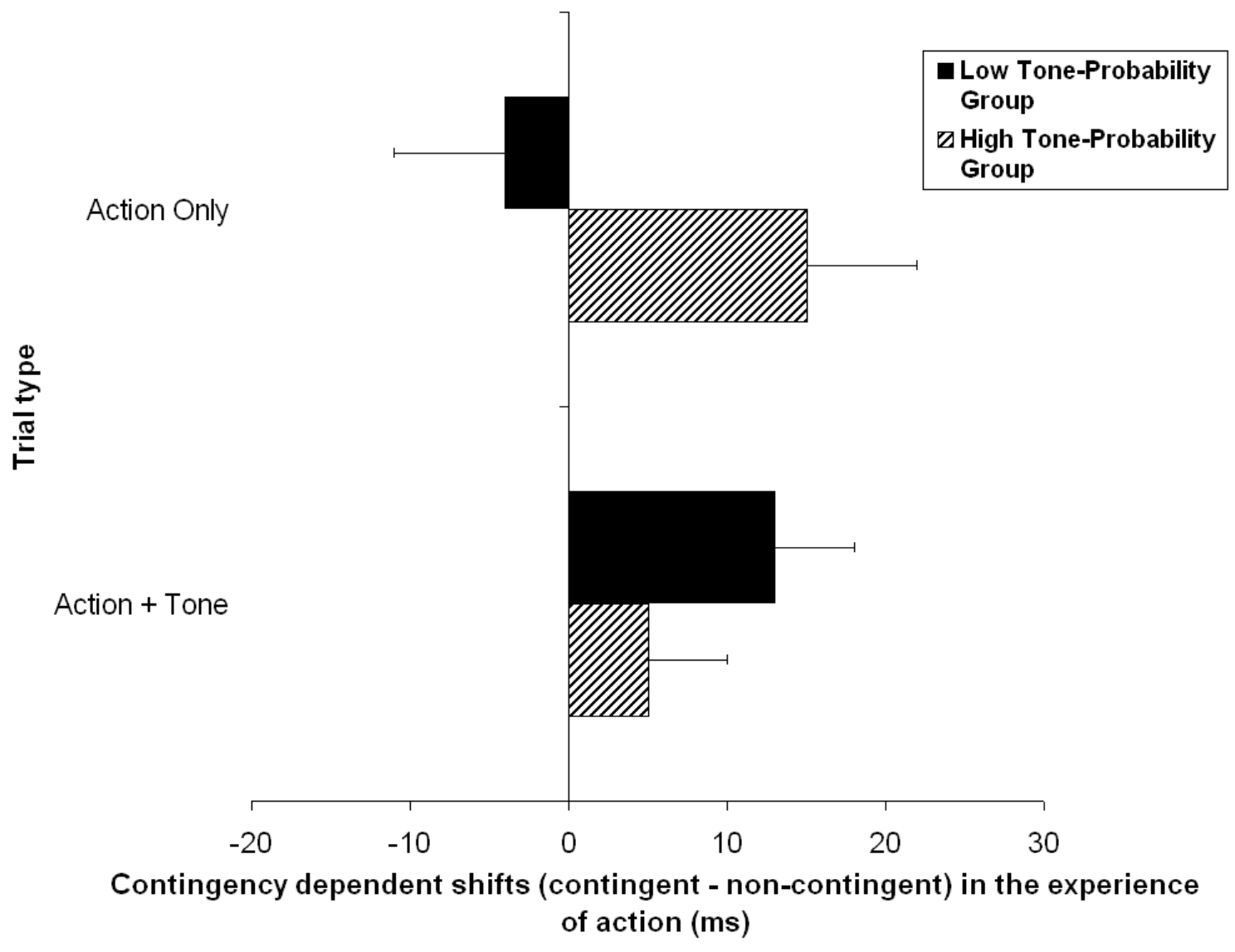

Figure 2. Effects of contingency on shifts in the experience of action (ms) for each toneprobability group, and for 'action only' and 'action + tone' trial types. Bars show standard error across subjects.

\section{Discussion}

This study systematically manipulated the contingency between voluntary actions and their effects, by varying the probability of a tone occurring in the absence of action. We also systematically assessed the contributions of prediction and postdictive inference to the experience of action, by measuring the shift in the subjective time of an action towards a subsequent tone in groups of participants for whom the probability of a tone following an action was either high or low. We show that both predictive and inferential components of action experience are sensitive to contingency, in the sense of $\Delta \mathrm{p}$. 
The importance of contingency in inferential causal learning is well known: contingency underlies operant behaviour in animals (Hammond, 1980), facilitation of reaction times by prior action-effect learning in humans (Elsner and Hommel, 2004), and also explicit causal judgements (Shanks \& Dickinson, 1991). Therefore, an influence of $\Delta \mathrm{p}$ on the inferential contribution to action experience might be expected. In contrast, the role of general contingency in predictive modulation of action experience is more striking. This result is at variance with purely motor theories of action awareness (Blakemore, Wolpert, $\&$ Frith, 2002; Berti et al. 2006). Significantly, we show that events entirely independent of the motor system have a strong influence on the experience of action. In particular, the occurrence of tones on trials where subjects had themselves chosen to make no action at all nevertheless influenced the subjective experience of action on other trials where they had decided to act. These trials in which subjects chose not to act carried information about the background probability of the tone in the absence of action. Our results show that subjects internalised this information, and that it influenced the subjective experience of their actions on trials with action. Purely motor theories (Blakemore, Wolpert \& Frith, 2002) link action awareness to predictions based on motor control signals occurring during action, and cannot easily explain this role of background context.

We suggest that the experience of action is not solely a result of direct conscious access to signals within the motor system. Rather, subjects form a causal model of the statistical relation between action and tone, which then structures their experience of their own action. Contingency learning makes a very general contribution, not only to causal knowledge, but also to consciousness. We averaged our estimates across several trials 
because of the high variability of human timing performance. Therefore, we could not measure the time-course of the learning process, but we can infer that causal learning occurs based on our contingency effects.

Our result does not prove that motor signals make no contribution to conscious experience of action. Indeed, a conscious urge to act can be generated by direct stimulation of motor areas in the absence of overt movement (Fried et al, 1991), and a conscious experience of being about to act can occur even when action itself is withheld (Libet et al., 1983; Brass \& Haggard, 2007). Rather, motoric contributions to the conscious experience of action may be embedded in a wider interpretative process that also includes background knowledge about external events.

The intentional binding paradigm offers a new measure of conscious experience of action, and specifically its relation to contingency. Previous research in this area referred to a 'causal impression' (Michotte, 1963), i.e., a directly-perceived feature of causal events. Our approach focuses on how experience of effects modulates the experience of the action that causes those effects. It therefore analyses the internal structure of causal relations in a way that impressionistic approaches are unable to do.

More generally, these findings clarify the relation between subjective time and causality. Hume (1739/1978) argued that the experience of when events happen underlies the perceived causal relation between them. Recent empirical research confirms this idea (Lagnado \& Sloman, 2006). We show that this relation also works in the reverse 
direction. The causal relations that we extract from the statistical regularities of our environment can determine the time at which we perceive individual events to occur, at least in the case of voluntary actions. We show that contingency modulates not just the perceived relation between action and effect, but the temporal perception of action itself. Our results further emphasize the intimate relationship between time perception and causality (Michotte, 1963), and between action and time perception (Yarrow et al., 2001). 


\section{$\underline{\text { References }}$}

Allan, L. G. (1980). A note on measurements of contingency between two binary variables in judgment tasks. Bulletin of the Psychonomic Society, 15, 147-149.

Berti, A., et al. (2006). Shared cortical anatomy for motor awareness and motor control. Science, 309, 488-491.

Blakemore, S-J, Wolpert, D. M., \& Frith, C. D. (2002). Abnormalities in the awareness of action. Trends in Cognitive Sciences, 6(6), 237-242.

Brass, M., \& Haggard, P. (2007). To do or not to do: the neural signature of self-control. Journal of Neuroscience, 27(34), 9141-9145.

Descartes, R. (1641/1979). Meditations on first philosophy, (D. A. Cress, Trans.). Indianapolis: Hackett Publishing.

Dickinson, A., \& Balleine, B. W. (2000). Causal cognition and goal-directed action. In C. Heyes \& L. Huber (Eds.), Evolution of cognition (pp. 185-204). Cambridge, MA: MIT Press.

Elsner, B., \& Hommel, B. (2004). Contiguity and contingency in the acquisition of action effects. Psychological Research, 68, 138-154.

Fried, I. Katz, A., McCarthy, G., Sass, K. J., Williamson, P., Spencer, S. S., \& Spencer, D. D. (1991). Functional organisation of human supplementary motor cortex studied by electrical stimulation. Journal of Neuroscience, 11, 3656-3666.

Haggard, P., Clark, S., \& Kalogeras, J. (2002). Voluntary action and conscious awareness. Nature Neuroscience, 5(4), 382-385. 
Hammond, L. J. (1980). The effect of contingency upon appetitive conditioning of free operant behaviour. Journal of the Experimental Analysis of Behaviour, 34, $297-$ 304.

Hume, D. (1739/1978). A treatise on human nature. Oxford: Clarendon Press.

James, W. (1890). The Principles of Psychology, London; MacMillan

Lagnado, D. A., \& Sloman, S. A. (2006). Time as a guide to cause. Journal of Experimental Psychology: Learning, Memory \& Cognition, 32, 451-460.

Libet, B., Gleason, C. A., Wright, E. W. \& Pearl, D. K. (1983). Time of conscious intention to act in relation to onset of cerebral activity (readiness potential): The unconscious initiation of a freely voluntary act. Brain, 106, 623-642.

Michotte, A. (1963). The perception of causality (T. R. Miles \& Elaine Miles, Trans.). New York: Basic Books.

Moore \& Haggard (2008). Awareness of action: Inference and prediction. Consciousness and Cognition. 17, 136-144.

Shanks, D. R., \& Dickinson, A. (1991). Instrumental judgements and performance under variations in action-outcome contingency and contiguity. Memory and Cognition, $19,353-360$.

Wegner, D. M. (2002). The Illusion of Conscious Will. Cambridge, MA: MIT Press.

Wundt, W. (1908). Grundzüge der physiologischen Psychologie. Vol. 1, 6th ed. Leipzig: Engelmann.

Yarrow, K., Haggard, P., Heal, R., Brown, P., \& Rothwell, J. C. (2001). Illusory perceptions of space and time preserve cross-saccadic perceptual continuity. Nature, 414, 302-305. 